\title{
Diagnostic value of PET-measured heterogeneity in myocardial blood flows during cold pressor testing for the identification of coronary vasomotor dysfunction
}

\author{
Thomas H. Schindler, MD, , ${ }^{\mathrm{a} b}$ Xiao-Li Zhang, MD, PhD, ${ }^{\mathrm{b}}$ Gabriella Vincenti, MD, ${ }^{\mathrm{a}}$ \\ Leila Mhiri, MD, ${ }^{\text {a }}$ Rene Nkoulou, MD, ${ }^{a}$ Hanjoerg Just, MD, ${ }^{c}$ Osman Ratib, MD, PhD, ${ }^{a}$ \\ Francois Mach, MD, ${ }^{a}$ Magnus Dahlbom, PhD, ${ }^{b}$ and Heinrich R. Schelbert, MD, PhD ${ }^{b}$
}

Background. We aimed to evaluate the diagnostic value of a positron emission tomography (PET)-measured heterogeneity in longitudinal myocardial blood flow (MBF) during cold pressor testing (CPT) and global MBF response to CPT from rest $(\triangle \mathrm{MBF})$ for identification of coronary vasomotor dysfunction.

Methods and Results. In 35 patients CPT-induced alterations in epicardial luminal area were determined with quantitative angiography as the reference. MBF was assessed over the whole left ventricle as global MBF and regionally in the mid and mid-distal myocardium as MBF difference or MBF heterogeneity with nitrogen-13 ammonia and PET. The sensitivity and specificity of a longitudinal MBF difference during CPT in the identification of epicardial vasomotor dysfunction were significantly higher than the global $\triangle \mathrm{MBF}$ to CPT $(88 \%$ vs $79 \%$ and $82 \%$ vs $64 \%$, respectively; $P<.05$ ). Combining both parameters resulted in an optimal sensitivity of $100 \%$ at the expense of an intermediate specificity of $73 \%$. The diagnostic accuracy was higher for the combined analysis than that for the MBF difference alone and global $\triangle M B F$ alone $(91 \%$ vs $86 \%$ and $74 \%$, respectively; $P<.05)$.

Conclusions. The combined evaluation of a CPT-induced heterogeneity in longitudinal MBF and the change in global MBF from rest may emerge as a new promising analytic approach to further optimize the identification and characterization of coronary vasomotor dysfunction. ( $J$ Nucl Cardiol 2007;14:688-97.)

Key Words: Blood flow $\bullet$ cold pressor test $\bullet$ coronary vasomotion $\bullet$ endothelium $\bullet$ positron emission tomography

Positron emission tomography (PET) measurements of global left ventricular (LV) myocardial blood flow (MBF) at rest and during sympathetic stimulation with cold pressor testing (CPT) are increasingly applied to

From the Department of Nuclear Cardiology, Cardiovascular Center, University Hospital of Geneva, Geneva, Switzerland; ${ }^{\mathrm{a}}$ Department of Molecular and Medical Pharmacology, David Geffen School of Medicine at UCLA, Los Angeles, Calif; ${ }^{\text {b }}$ Medical Center, University Hospital Freiburg, Freiburg, Germany. ${ }^{\mathrm{c}}$

This work was supported in part by National Institutes of Health grant HL 33177 from the National Heart, Lung, and Blood Institute and by a grant from the government of Baden-Wuerttemberg, Germany, for the Center of Clinical Research II: Cardiovascular Diseases: Analysis and Integration of Form and Function at Albert-LudwigUniversity Freiburg (project THS-A1/A2).

Received for publication May 6, 2007; final revision accepted June 25, 2007.

Reprint requests: Thomas H. Schindler, MD, Cardiologie Nucléaire, Service Cardiologie, Département de Médecine Interne, Hôpitaux Universitaire de Genève, Rue Micheli-du-Crest 24, 1211 Genève 14, Switzerland; thomas.schindler@hcuge.ch. $1071-3581 / \$ 32.00$

Copyright $₫ 2007$ by the American Society of Nuclear Cardiology. doi:10.1016/j.nuclcard.2007.06.120 assess endothelium-related coronary vasomotor function. ${ }^{1-8}$ Such noninvasively obtained information on endothelium-dependent coronary vasomotor function is considered to carry important diagnostic and prognostic information..$^{9-12}$ Of particular interest are recent findings regarding the effects of medical preventive intervention on vasomotor dysfunction in the peripheral circulation in patients with acute coronary syndrome and in hypertensive postmenopausal women. ${ }^{13,14}$ In these patients the institution of medical therapy led to an improvement in endothelium-dependent vasomotor function, which was also associated with an improved cardiovascular outcome, but not in those individuals in whom improvement failed to occur. Conceptually, primary or secondary preventive medical intervention, with regard to the development and progression of coronary artery disease (CAD), could be successfully monitored according to the findings of abnormal coronary vasomotor function in response to $\mathrm{CPT}^{1,15,16}$ or to pharmacologic vasodilation (or both). ${ }^{8,17-19}$ Global LV MBF responses to CPT in the individual, however, may underlie some variability resulting from daily temporal fluctuations of coronary 
Table 1. Characteristics of study population

\section{Control group}

No. of patients

Age (y)

$\operatorname{Sex}(F / M)(n)$

Body mass index $\left(\mathrm{kg} / \mathrm{m}^{2}\right)$

Hypertension (n)

Hypercholesterolemia (n)

Smoking (n)

Fasting plasma concentrations

Total cholesterol $(\mathrm{mg} / \mathrm{dL})$

LDL cholesterol $(\mathrm{mg} / \mathrm{dL})$

HDL cholesterol $(\mathrm{mg} / \mathrm{dL})$

Triglycerides $(\mathrm{mg} / \mathrm{dL})$

Glucose $(\mathrm{mg} / \mathrm{dL})$

Epicardial LA $\left(\mathrm{mm}^{2}\right)$

At rest

During CPT

Change to CPT

Global MBF $\left(\mathrm{mL} \cdot \mathrm{g}^{-1} \cdot \mathrm{min}^{-1}\right)$

At rest

During CPT

Change to CPT

MBF difference $\left(\mathrm{mL} \cdot \mathrm{g}^{-1} \cdot \mathrm{min}^{-1}\right)$

At rest

During CPT

$\begin{array}{cc}11 & 24 \\ 58 \pm 7 & 57 \pm 10 \\ 7 / 4 & 16 / 8 \\ 24 \pm 4 & 25 \pm 3 \\ 0 & 10 \\ 0 & 8 \\ 0 & 6 \\ 164 \pm 33 & 222 \pm 35^{*} \\ 96 \pm 25 & 152 \pm 25^{*} \\ 56 \pm 9 & 52 \pm 13 \\ 120 \pm 30 & 138 \pm 50^{*} \\ 86 \pm 6 & 98 \pm 14 \\ 5.2 \pm 1.3 & \\ 6.1 \pm 1.0 & 5.5 \pm 1.1 \\ 0.88 \pm 0.36 & 4.5 \pm 0.9^{*} \\ 0.61 \pm 0.17 & 1.02 \pm 0.85^{*} \\ 0.90 \pm 0.16 & \\ 0.29 \pm 0.10 & 0.57 \pm 0.13 \\ 0.001 \pm 0.05 & 0.63 \pm 0.25^{*} \\ 0.05 \pm 0.02 & 0.05 \pm 0.21^{*} \\ & \\ & \pm .001 \pm 0.07 \\ & \pm .15 \pm 0.08^{*}\end{array}$

At-risk group

Values are given as mean \pm SD, unless otherwise indicated. $H D L$, High-density lipoprotein.

${ }^{*} P<.0001$ versus control group.

circulatory (dys)function or interindividual differences in hemodynamic responses to CPT..$^{1,3-5,20-23}$ Thus, although group responses of global LV MBFs to CPT in persons with coronary risk factors are commonly reduced, a clear identification of coronary vasomotor dysfunction in the individual person sometimes may remain uncertain.

Emerging evidence suggests that the assessment of a longitudinal, base-to-apex decrease in myocardial perfusion or MBF during pharmacologically stimulated hyperemia or during CPT may identify an impairment of flow-mediated and, thus, endothelium-dependent coronary vasodilation. ${ }^{24-27}$ With this in mind, we hypothesized that, apart from the conventional evaluation of CPT-induced change in global MBF ( $\triangle \mathrm{MBF}$ to $\mathrm{CPT})$, the additional analysis of a heterogeneity in longitudinal MBF during sympathetic stress ${ }^{27}$ could further improve the noninvasive identification of coronary vasomotor dysfunction.

Accordingly, we aimed to determine the diagnostic value of a PET-measured heterogeneity in longitudinal $\mathrm{MBF}$ during CPT and the global $\triangle \mathrm{MBF}$ to CPT for the identification of coronary vasomotor dysfunction in individuals with coronary risk factors but with normal coronary angiograms.

\section{METHODS}

\section{Patient population}

We studied 35 patients (12 men and 23 women; mean age, $57 \pm 9$ years) without angiographic evidence of CAD (Table 1). They were classified into 2 groups according to presence or absence of various coronary risk factors. Twenty-five study participants with coronary risk factors were assigned to the "at-risk" group, whereas eleven age-matched healthy individuals without traditional coronary risk factors served as the control group. In the at-risk group, 10 patients had hypertension ( $\geq 140 / 90 \mathrm{~mm} \mathrm{Hg}$ ), 8 had hypercholesterolemia (total cholesterol $\geq 240 \mathrm{mg} / \mathrm{dL}$, low-density lipoprotein [LDL] cholesterol $\geq 160 \mathrm{mg} / \mathrm{dL}$ ), and 6 were chronic smokers ( $>10$ pack-years). Quantitative coronary angiography (QCA) at baseline and during CPT to establish flow-mediated vasoreactivity of the epicardial coronary artery was performed as described previ- 
A

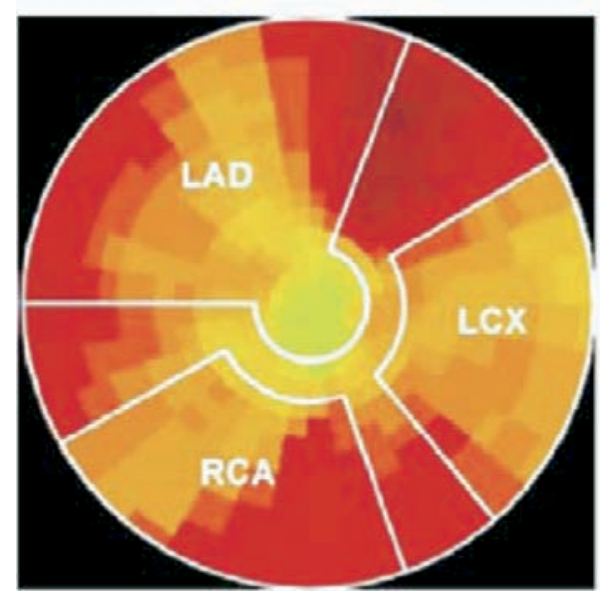

B

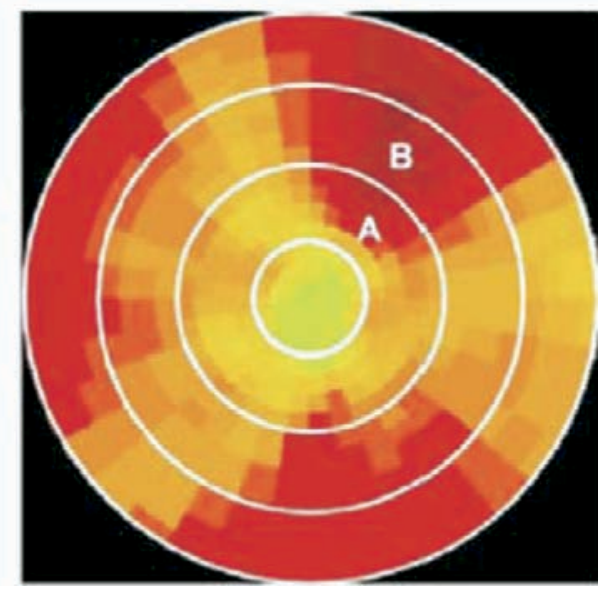

Figure 1. Polar maps of MBFs and assignment of ROIs. A, Three coronary artery territories. B, Circumferential ROIs for mid $(B)$ and mid-distal $(A)$ portion of left ventricle. $L A D$, Left anterior descending artery; $L C x$, left circumflex artery; $R C A$, right coronary artery.

ously. ${ }^{28}$ Within 20 days of coronary angiography, regional $\mathrm{MBF}$ at rest and in response to CPT was measured in each participant in milliliters per gram per minute with nitrogen-13 ammonia and PET. ${ }^{2,29}$ All study participants had normal wall motion on angiographic evaluation. Each patient was screened by a complete history, physical examination, and blood chemistry. Exclusion criteria included a history of cardiovascular, liver, renal, endocrine, or inflammatory disease. Only individuals not taking vasoactive medication, such as angiotensinconverting enzyme inhibitors, calcium channel blockers, or statins, were recruited. All smokers refrained from smoking for at least 12 hours before QCA and PET studies. Routine blood chemistry analysis included total cholesterol, high-density lipoprotein and LDL cholesterol, very low-density lipoprotein cholesterol, triglycerides, and glucose. The study was approved by the local ethics committee of the University of Freiburg (Freiburg, Germany), and written informed consent was obtained from all individuals.

\section{QCA}

All patients underwent routine diagnostic coronary angiography for evaluation of chest pain via a biplane, isocentric multidirectional digital angiographic system (BICOR-HICOR; Siemens, Erlangen, Germany). ${ }^{28}$ End-diastolic images of coronary arteries were evaluated quantitatively with automatic contour detection as described previously. ${ }^{28}$ In all individuals with normal coronary angiograms, as defined by smooth luminal surface of the coronary vessel without diffuse diameter reduction or stenoses, quantitative biplane measurements were obtained in a selected, distinct 4- to 8-mm-long, relatively straight proximal left anterior descending coronary artery segment $(\mathrm{n}=18)$ or left circumflex coronary artery segment ( $\mathrm{n}$ $=17$ ). Estimation of the luminal area (LA) assumed an elliptical shape at baseline and during $\mathrm{CPT} .{ }^{28,30}$ Calculation of the radiologic magnification factor of the measured segment was used for scaling of the data from pixels to millimeters. ${ }^{28}$ Thus the epicardial LA was determined at baseline and during CPT and the epicardial vasomotor response quantified.

\section{Evaluation and measurement of MBF}

PET. MBF was measured noninvasively by use of intravenous N-13 ammonia, serial image acquisition with PET (ECAT EXACT HR+; CTI/Siemens, Knoxville, Tenn), and a 2-compartment tracer kinetic model, as described previously. ${ }^{29}$ Transmission images were recorded first for 20 minutes. Beginning with each intravenous $\mathrm{N}-13$ ammonia injection (555-740 MBq), serial transaxial, attenuation-corrected images were acquired for 19 minutes (16 frames, with $12 \times 10$ seconds, $2 \times 30$ seconds, $1 \times 60$ seconds, and $1 \times 15$ minutes $)$. MBF measurements were performed at baseline and during CPT. For CPT, study participants immersed the left hand in ice water for 60 seconds, and N-13 ammonia was injected again while CPT continued for another 60 seconds. Between MBF measurements, we allowed 45 minutes for physical decay of N-13 ammonia. From the last 15-minute transaxial image, reoriented short- and long-axis myocardial slices and the corresponding polar map were submitted to visual and semiquantitative analysis. ${ }^{2}$ The interobserver and intraobserver reproducibility of PET-measured MBFs at rest and during CPT has been reported recently. ${ }^{31,32}$ Heart rate, blood pressure, and a 12-lead electrocardiogram were recorded continuously during each MBF measurement. From the average of heart rate and blood pressure during the first 2 minutes of each image acquisition, the rate-pressure product (RPP) was determined as an index of cardiac work.

Quantitative evaluation of MBF. On the polar map of the last 15-minute image set, regions of interest (ROIs) were assigned to myocardial territories of the 3 coronary arteries 


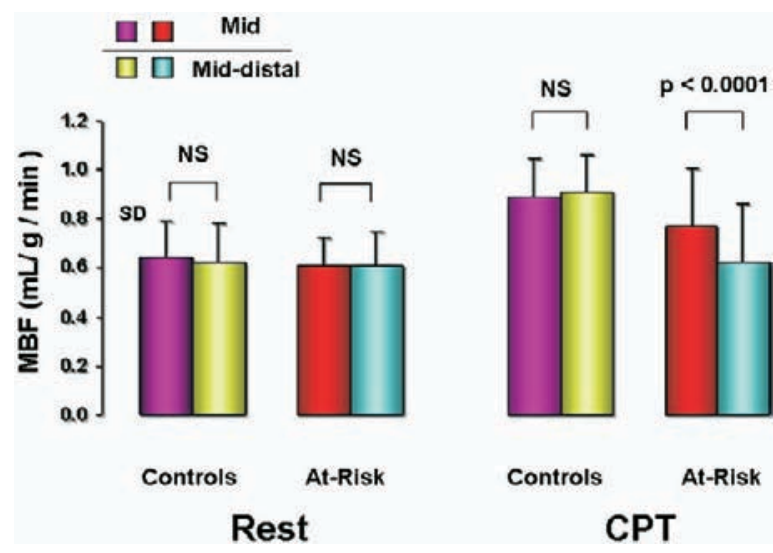

Figure 2. MBFs at rest and during CPT for mid and mid-distal portions of LV myocardium in both study groups.

(Figure 1). As shown in Figure 1, 2 circumferential ROIs were assigned to the mid and mid-distal portion of the left ventricle. In addition, a $25-\mathrm{mm}^{2}$ ROI was assigned to the LV blood pool on the most basal short-axis slice for deriving the arterial tracer input function. The ROIs were then copied to the serial polar maps acquired during the first 2 minutes after tracer injection. The time-activity curves derived from these ROIs were fitted with a 2-compartment tracer kinetic model, and regional MBF values in milliliters per gram per minute were obtained. ${ }^{29}$ MBFs in the 3 coronary artery territories were averaged, and mean global MBF was derived. Changes in MBF from rest to $\mathrm{CPT}$ were defined in milliliters per gram per minute as global $\triangle \mathrm{MBF}$. Furthermore, a decrease in MBF from mid to middistal LV myocardium was defined as MBF difference (in milliliters per gram per minute) or heterogeneity in longitudinal $\mathrm{MBF}$ as indicative of a perfusion gradient. ${ }^{27}$

\section{Data analysis}

Data are presented as mean $\pm \mathrm{SD}$ for quantitative and absolute frequencies for qualitative variables. For comparison of differences, appropriate Wilcoxon rank sum tests for independent or paired samples were used (SAS Institute, Cary, NC). A comparison of CPT-induced change in global MBF and MBF difference between the different groups was performed by 2-way analysis of variance (ANOVA), followed by the Scheffé multiple comparisons test. Correlations between selected variables were estimated by Spearman correlation coefficients. Sensitivities, specificities, and diagnostic accuracies were calculated. All test procedures were 2-sided, with $P \leq .05$ indicating statistical significance.

\section{RESULTS}

\section{Clinical characteristics}

Table 1 summarizes the characteristics of the 2 study groups. Total cholesterol, LDL cholesterol, and triglyceride levels were significantly higher in the at-risk group than in the control group, whereas high-density lipoprotein cholesterol tended to be lower. Glucose levels and body mass index did not differ significantly between the study groups.

\section{Hemodynamic parameters}

Hemodynamic parameters during angiographic assessment of epicardial coronary vasomotion and during PET measurements of MBF at baseline and during CPT are listed in Table 2. The RPP (heart rate $\times$ systolic blood pressure) was used as an index of cardiac work and as a measure of the effectiveness of sympathetic stimulation with CPT. Heart rate and blood pressure were comparable at rest and during CPT between QCA and PET studies. CPT induced a significant increase in heart rate and systolic and diastolic blood pressure. Consequently, the RPP increased significantly from rest to CPT. Notably, there was no significant difference between the RPP at rest and during CPT at the time of the QCA and of the PET study. In addition, when the change in the RPP during CPT from rest was evaluated, no significant difference was found between QCA and PET evaluation of $\mathrm{MBF}$, indicating comparable myocardial workload on both study days.

\section{Findings on quantitative angiography}

Table 1 denotes the mean changes in epicardial LA to CPT in the control group and in the at-risk group. At baseline, the mean epicardial LA did not differ significantly between the groups. In the at-risk group the LA abnormally decreased from $5.5 \pm 1.1 \mathrm{~mm}^{2}$ to $4.5 \pm 0.9$ $\mathrm{mm}^{2}$ by CPT $(P<.0001)$, reflecting a mean change in LA $(\Delta \mathrm{LA})$ of $-1.02 \pm 0.85 \mathrm{~mm}^{2}$. Conversely, the control group showed a significant flow-mediated increase in LA from $5.2 \pm 1.3 \mathrm{~mm}^{2}$ to $6.1 \pm 1.0 \mathrm{~mm}^{2}(P$ $<.0001)$, which represented a mean $\Delta$ LA of $0.88 \pm 0.36$ $\mathrm{mm}^{2}$. The group comparison of a CPT-induced decrease in mean LA in the at-risk group was significant when compared with a blood flow-mediated increase in the mean LA in the control group $(P<.0001$ by ANOVA).

\section{MBF response to CPT}

Quantitative assessment of global MBF at rest was comparable between the study groups (Table 1). Global MBF during CPT in the at-risk group was significantly lower than in the control group. Thus the change in mean MBF to CPT from rest $(\triangle \mathrm{MBF})$ was significantly impaired in the at-risk group when compared with the control group (Table 1). The group comparison of $\triangle \mathrm{MBF}$ to $\mathrm{CPT}$ in the at-risk group was 
Table 2. Hemodynamics in QCA and PET studies at rest and during CPT

\begin{tabular}{|c|c|c|c|c|c|c|}
\hline \multirow[b]{2}{*}{ Test } & \multicolumn{3}{|c|}{ QCA } & \multicolumn{3}{|c|}{ PET } \\
\hline & Rest & CPT & $\begin{array}{l}\text { Change } \\
\text { from rest } \\
\text { to CPT }\end{array}$ & Rest & CPT & $\begin{array}{l}\text { Change } \\
\text { from rest } \\
\text { to CPT }\end{array}$ \\
\hline HR (beats/min) & $62 \pm 7$ & $67 \pm 7^{*}$ & & $62 \pm 7$ & $66 \pm 7^{*}$ & \\
\hline $\mathrm{SBP}(\mathrm{mm} \mathrm{Hg})$ & $128 \pm 16$ & $152 \pm 18^{*}$ & & $130 \pm 15$ & $149 \pm 16^{*}$ & \\
\hline DBP (mm Hg) & $73 \pm 8$ & $77 \pm 8^{*}$ & & $72 \pm 7$ & $77 \pm 7^{*}$ & \\
\hline $\mathrm{RPP}(\mathrm{mm} \mathrm{Hg} / \mathrm{min})$ & $8,096 \pm 1,348$ & $10,183 \pm 1,574^{*}$ & $2,086 \pm 778^{*}$ & $8,056 \pm 1,380$ & $9,880 \pm 1,476^{*}$ & $1,823 \pm 1,012^{*}$ \\
\hline MAP (mm Hg) & $92 \pm 9$ & $102 \pm 9^{*}$ & & $91 \pm 9$ & $101 \pm 8^{*}$ & \\
\hline
\end{tabular}

Values are given as mean $\pm \mathrm{SD}$.

$H R$, Heart rate; SBP, systolic blood pressure; $D B P$, diastolic blood pressure; $M A P$, mean arterial blood pressure.

${ }^{*} P \leq .0001$ for CPT versus rest for each corresponding hemodynamic parameter in QCA and PET study evaluation, respectively. $P=$ NS for each corresponding hemodynamic parameter between QCA and PET study evaluation.

significant when compared with the control group $(P$ $\leq .0001$ by ANOVA).

The comparison of regional MBFs at rest in the mid and mid-distal direction demonstrated similar values for the mid and mid-distal LV sections in both groups (0.64 \pm 0.15 and $0.62 \pm 0.16 \mathrm{~mL} \cdot \mathrm{g}^{-1} \cdot \mathrm{min}^{-1}$ in control group and $0.61 \pm 0.11$ and $0.61 \pm 0.14$ $\mathrm{mL} \cdot \mathrm{g}^{-1} \cdot \min ^{-1}$ in at-risk group, respectively; $P=$ not significant [NS]) (Figure 2). In the control group CPT induced a homogeneous increase in MBF with comparable mid and mid-distal LV MBFs $(0.89 \pm 0.16$ and $0.92 \pm 0.15 \mathrm{~mL} \cdot \mathrm{g}^{-1} \cdot \mathrm{min}^{-1}$, respectively; $P=\mathrm{NS}$ ) (Figure 2). In the at-risk group, however, the MBF response to CPT in the mid LV was significantly lower than those in the control group $(P<.001)$. Moreover, sympathetic stimulation with CPT resulted in MBF heterogeneities in the at-risk group. MBF increased to $0.77 \pm 0.24 \mathrm{~mL} \cdot \mathrm{g}^{-1} \cdot \mathrm{min}^{-1}$ in the mid LV myocardium but only to $0.62 \pm 0.24 \mathrm{~mL} \cdot \mathrm{g}^{-1} \cdot \mathrm{min}^{-1}$ in the mid-distal LV myocardium (Figure 2). This resulted in an MBF difference of $0.15 \pm 0.08 \mathrm{~mL} \cdot \mathrm{g}^{-1} \cdot \mathrm{min}^{-1}$ during CPT between the mid and mid-distal LV myocardium (Table 1). No such difference between the mid and mid-distal LV MBF was observed in the control group $\left(0.05 \pm 0.02 \mathrm{~mL} \cdot \mathrm{g}^{-1} \cdot \min ^{-1}, P=\mathrm{NS}\right)$ (Table 1$)$. The group comparison of the CPT-induced MBF difference between the at-risk and control groups was significantly different $(P<.0001$ by ANOVA). Moreover, the CPTinduced MBF differences in the 2 coronary territories supplied by the vessel not evaluated on quantitative angiography in the at-risk group were similar to the MBF differences in coronary territories subtended by coronary vessels which were submitted to quantitative analysis. The MBF differences during CPT were comparable $(0.13$ \pm 0.12 vs $\left.0.15 \pm 0.08 \mathrm{~mL} \cdot \mathrm{g}^{-1} \cdot \min ^{-1}, P=\mathrm{NS}\right)$,

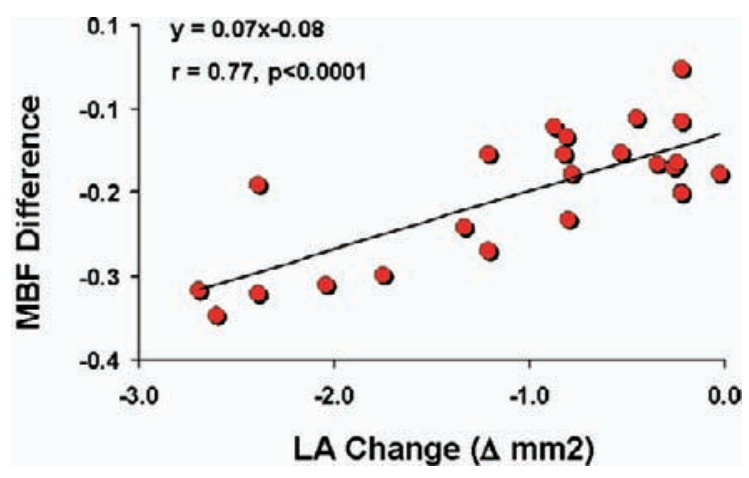

Figure 3. Correlation between $\mathrm{MBF}$ difference and $\triangle \mathrm{LA}$ during CPT.

suggesting that the MBF difference in at-risk individuals occurs homogeneously in the entire coronary circulation.

\section{Correlation between CPT-induced changes in epicardial LA and MBF gradient}

To evaluate a possible association between CPTinduced alterations of the epicardial artery and the relative decrease in MBF from the mid to mid-distal LV myocardium, the change in epicardial LA was compared with the MBF difference during CPT. For the entire study group, there was a significant correlation between the change in epicardial LA ( $\triangle \mathrm{LA})$ to CPT and the MBF difference during CPT between the mid and mid-distal LV myocardium $(r=0.90, P<.0001)$. When the at-risk group alone was examined, as shown in Figure 3, the CPT-induced decrease in epicardial LA and the MBF difference were also significantly correlated $(r=0.77$, $P<.0001)$. These findings emphasize that the MBF difference is indeed related to functional alterations of the epicardial conduit vessels during CPT. 
Table 3. Diagnostic accuracy of PET-measured MBF alterations during CPT in detection of coronary vasomotor function as determined by QCA

\begin{tabular}{lccc}
\hline & $\boldsymbol{\Delta}$ MBF to CPT & $\begin{array}{c}\text { MBF difference } \\
\text { during CPT }\end{array}$ & Combined \\
\hline Sensitivity & $19 / 24(79)$ & $21 / 24(88)$ & $24 / 24(100)$ \\
Specificity & $7 / 11(64)$ & $9 / 11(82)$ & $8 / 11(73)$ \\
PPV & $19 / 23(83)$ & $21 / 23(91)$ & $24 / 27(88)$ \\
NPV & $7 / 12(58)$ & $9 / 12(75)$ & $8 / 8(100)$ \\
Diagnostic accuracy & $26 / 35(74)$ & $30 / 35(86)$ & $32 / 35(91)$ \\
\hline
\end{tabular}

Values are given as $\mathrm{n}(\%)$.

PPV, Positive predictive value; NPV, negative predictive value.

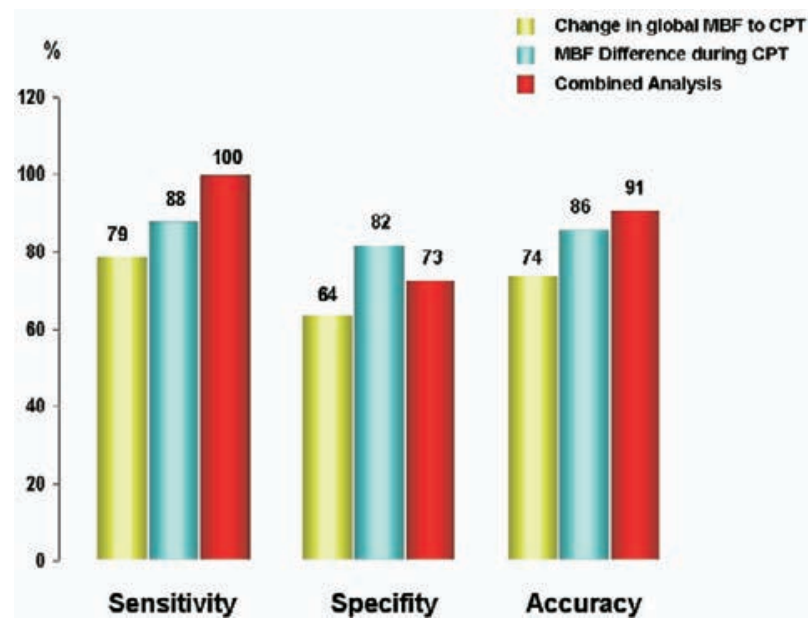

Figure 4. Sensitivity, specificity, and diagnostic accuracy of PET-measured MBF alterations during CPT in identification of epicardial vasomotor dysfunction.

\section{Diagnostic accuracy of global $\triangle M B F$ to CPT and CPT-related MBF difference in detection of coronary vasomotor dysfunction}

The receiver operating characteristic curve (ROC) for the detection of epicardial vasomotor dysfunction by the MBF difference during $\mathrm{CPT}$ and global $\triangle \mathrm{MBF}$ to CPT yielded an optimal cutoff point of greater than 0.071 and less than or equal to $0.216 \mathrm{~mL} \cdot \mathrm{g}^{-1} \cdot \mathrm{min}^{-1}$, respectively. By use of this ROC-defined threshold, the sensitivity, specificity, negative predictive value, positive predictive value, and diagnostic accuracy of MBF difference during CPT, global $\triangle \mathrm{MBF}$ to CPT, and the combined analysis of both parameters for the detection of coronary vasomotor dysfunction are given in Table 3 and Figure 4. The sensitivity of the MBF difference to identify abnormal epicardial vasomotion was significantly higher than with the change in global MBF from rest to CPT ( $\triangle \mathrm{MBF})(88 \%$ vs $79 \%, P<.05)$ (Figure 4$)$, whereas combining both parameters resulted in an opti- mal sensitivity of $100 \%$. The specificity was high for the MBF difference and relatively low for $\triangle \mathrm{MBF}$ (82\% vs $64 \%, P<.05)$. Applying both parameters resulted in an intermediate specificity of $73 \%$ that was significantly lower than that for the MBF difference, at $82 \%(P<$ $.05)$, though still higher than that for $\triangle \mathrm{MBF}$, at $64 \%$, but not significantly. The diagnostic accuracy of the CPTrelated MBF difference for evaluation of coronary vasomotor dysfunction was not significantly higher as compared with $\triangle \mathrm{MBF}$ to $\mathrm{CPT}$ ( $86 \%$ vs $74 \%$ ). The combined analysis of both parameters, however, yielded the highest diagnostic accuracy, which was significantly higher than that for the MBF difference alone and $\triangle \mathrm{MBF}$ alone (91\% vs $86 \%$ and $74 \%$, respectively; $P<.05$ ).

\section{DISCUSSION}

This study is unique in that it demonstrates a close association between CPT-induced alterations in the LA of the epicardial artery and a heterogeneous response of regional MBF from the mid to mid-distal LV myocardium, as indicative for an MBF gradient. This finding provides direct angiographic evidence that functional alterations, such as a sympathetically mediated paradoxical vasoconstriction of the epicardial artery, may indeed account for a heterogeneity in longitudinal MBF during $\mathrm{CPT}$, as also observed in previous investigations with PET. ${ }^{24-27}$ In particular, the assessment of a CPT-related heterogeneity in longitudinal MBF appears to be superior to the change in global MBF to CPT from rest $(\triangle \mathrm{MBF})$ in identifying coronary vasomotor dysfunction. The combined analysis of both parameters, however, yielded the highest diagnostic accuracy for the noninvasive detection of coronary vasomotor dysfunction. Thus the additional evaluation of a heterogeneity in longitudinal MBF during CPT, apart from the conventional global $\triangle \mathrm{MBF}$ to $\mathrm{CPT}$, may emerge as another reliable parameter to optimize the identification and characterization of coronary vasomotor dysfunction. 


\section{Coronary vasomotor function and heterogeneity in longitudinal MBF}

The regulation and modulation of the coronary blood flow underlies a complex metabolic and autonomic control to meet the nutrition and oxygen requirements of the heart. ${ }^{33,34}$ Whereas the epicardial coronary arteries mediate, more or less, a conductance function, the coronary arteriolar vessels predominantly determine the coronary vascular resistance and, thus, the increase in MBF during times of increases in metabolic oxygen demand. ${ }^{33}$ Notably, the integrity of the coronary arteriolar resistance vessels reflects an important determinant in mediating antiatherosclerotic effects. ${ }^{9,10}$ This is because increases in coronary flow resulting from a metabolically induced decrease in vascular resistance of the coronary arteriolar vessels lead to a flow-mediated vasodilation via endothelium-dependent release of nitric oxide, which also implicates nitric oxide-related antithrombotic and antiatherosclerotic effects. ${ }^{35-37}$ Coronary flow increases during pharmacologically induced hyperemia or during sympathetic stimulation with CPT, therefore, may be seen as a cumulative assessment of both the epicardial coronary arteries and the coronary arteriolar resistance vessels, rather than a reflection of the role of the coronary microcirculation alone. ${ }^{38}$ Early CAD-related functional or structural alterations of the arterial wall, however, may disturb the flow-mediated coronary vasodilatory capacity. ${ }^{33,38}$ Regarding the latter, diffuse luminal narrowing or functional alterations of the epicardial coronary arteries, ${ }^{24,26,39}$ associated with an impairment of flow-mediated coronary vasodilatory function, have been proposed to account for a previously observed longitudinal, base-to-apex myocardial perfusion gradient or a heterogeneity in LV MBF during pharmacologically stimulated hyperemia in patients with diffuse CAD or with coronary risk factors. ${ }^{19,24,26,40}$ The mechanism underlying an MBF heterogeneity during hyperemic flow increases may be best described by the Hagen-Poiseuille equation. ${ }^{26,39,41}$ Accordingly, the resistance to flow depends on the length of the tube and the flow velocity and, importantly, inversely on the fourth power of the vessel diameter. ${ }^{24,41}$ Normally, increases in intracoronary flow velocity induce a flow-mediated vasodilation of the coronary artery that compensates for the velocity-related increase in coronary resistance to keep the resistance low. ${ }^{42,43}$ Conversely, an impairment of the flow-mediated vasodilation, as a result of the presence of endothelial dysfunction or CAD-induced diffuse epicardial luminal narrowing, may impede the dilation of the coronary artery during higher coronary flows. ${ }^{30,43}$ As coronary angiographic investigations have shown, ${ }^{39}$ an impairment of a flow-mediated coronary vasodilation leads to an increase in intravascular resistance during hyperemic flows with a progressive decline in intracoronary pressure along the coronary artery. This progressive proximal-to-distal decline in intracoronary pressure during hyperemic coronary flow increases ${ }^{39}$ has been put forward as a cause for the perfusion or MBF heterogeneity during pharmacologic vasodilation. ${ }^{24,26,39}$ Direct confirmation through comparative studies between PET flow measurements and quantitative angiography is still missing or is incomplete. A recently performed investigation provides some initial evidence of an association between the manifestation of a myocardial perfusion heterogeneity during dipyridamole-stimulated flow increases and the presence of CAD-induced diffuse arterial narrowing. ${ }^{24}$ As our study and recent investigations demonstrate, ${ }^{27}$ a heterogeneity in longitudinal MBF may also occur during sympathetic stimulation with CPT in individuals with coronary risk factors. Importantly, the current observations indicate that a heterogeneity in longitudinal MBF during CPT is indeed related to sympathetically mediated functional alterations of the epicardial artery. Thus these findings and previous investigations ${ }^{19,26,27}$ support the evolving concept, as raised first by Gould et $a l,{ }^{24}$ that the assessment of a myocardial perfusion gradient or a longitudinal heterogeneity in MBF by means of PET could serve as an important tool by which to noninvasively identify early functional or structural alterations of the epicardial coronary artery. ${ }^{24,26,27,40}$ Such a noninvasive measure of the early stages of the development of CAD may also provide important predictive information on future cardiovascular events. ${ }^{18,19}$

The endothelium-related global $\triangle \mathrm{MBF}$ to $\mathrm{CPT}$ in healthy individuals has been reported to range between $30 \%$ and $50 \%$ and, thus, reveals some variability. ${ }^{1-5}$ The reason for this variability is uncertain and may be related to differences in daily temporal fluctuations of coronary circulatory (dys)function or interindividual differences in hemodynamic responses to CPT (or both). ${ }^{1,3-5,20-23,44}$ Furthermore, there is also an intrinsic variability of the severity of endothelium-dependent vasomotor dysfunction in the individual, despite the presence of a similar risk profile, which has to be taken into consideration. ${ }^{11,37}$ Another explanation is that the extent of a diminished global MBF response to CPT, as indicative for coronary endothelial dysfunction, ${ }^{45}$ is also determined by an $\alpha$-adrenergically mediated constriction of the vascular smooth muscle cells, which is closely related to the degree of sympathetic stimulation during CPT, as reported recently. ${ }^{27}$ Thus interindividual differences in sympathetic stimulation of the vascular smooth muscle cells are likely to add some variability to the abnormal global MBF responses during CPT. Notably, the assessment of a heterogeneity in longitudinal MBF during pharmacologically stimulated hyperemia or during CPT 
may evolve as another promising quantitative index by which to identify an impairment of flow-mediated and, thus, endothelium-dependent coronary vasodilation. ${ }^{26,27}$ Such an approach might also overcome the aforementioned limitations of the evaluation of the global $\triangle \mathrm{MBF}$ to CPT and, thereby, could lead to a further improvement in the diagnostic accuracy of the detection of coronary vasomotor dysfunction. Thresholds for a heterogeneity in longitudinal MBF during CPT and a diminished increase in global $\triangle \mathrm{MBF}$ to $\mathrm{CPT}$ were derived from an ROC analysis and validated in this group of patients who underwent coronary angiography within 20 days before the PET study. In this validation study abnormal endothelium-dependent vasomotion was defined as an absent vasodilation or vasoconstriction of the epicardial artery during CPT. ${ }^{30,46}$ Such an impairment of epicardial vasomotor function during CPT has been demonstrated to extend to the site of the coronary arteriolar resistance vessels. ${ }^{1,3,5}$ Using the epicardial vasomotor response to $\mathrm{CPT}$ as a reference, we performed an ROC analysis to define the optimal threshold for the identification of coronary vasomotor dysfunction by a heterogeneity in longitudinal MBF during CPT and the global $\triangle \mathrm{MBF}$ to $\mathrm{CPT}$. This analysis relied exclusively on the heterogeneity in longitudinal MBF during CPT and on CPT-induced global $\triangle \mathrm{MBF}$ with an ROC-defined threshold of greater than 0.071 and less than or equal to 0.216 $\mathrm{mL} \cdot \mathrm{g}^{-1} \cdot \min ^{-1}$, respectively, to distinguish between abnormal and normal coronary vasomotor function. By use of these thresholds, the sensitivity and specificity of the MBF heterogeneity during CPT were significantly higher as compared with the global $\triangle \mathrm{MBF}$ to CPT ( $88 \%$ vs $79 \%$ and $82 \%$ vs $64 \%$, respectively). This also resulted in a higher diagnostic accuracy to detect coronary vasomotor dysfunction, with $86 \%$ for the heterogeneity in longitudinal MBF as compared with $74 \%$ for global $\triangle \mathrm{MBF}$ to CPT. Furthermore, when we integrated both quantitative measures from the PET flow study, considering the vasomotor abnormality to be significant when detected by one of the two approaches, the diagnostic accuracy was significantly increased to $91 \%$. In this regard, the sensitivity increased to $100 \%$ at the expense of a lower specificity of $73 \%$. Thus our results indicate that the assessment of a heterogeneity in longitudinal MBF may be more sensitive in identifying coronary vasomotor dysfunction than the global $\triangle \mathrm{MBF}$ to CPT. Nevertheless, the combined analysis of both parameters yielded the highest accuracy in the evaluation of coronary vasomotor function as compared with the separate analysis of the MBF heterogeneity during CPT or the global MBF response to CPT. It follows, then, that combining both quantitative measures of endotheliumrelated changes in MBF to CPT could be the preferred approach in the evaluation of coronary vasomotor function.

\section{Limitations}

There are shortcomings of this study worthy to be considered in the interpretation of the data. First, coronary intravascular ultrasound was not used in our study protocol. Thus we cannot rule out the presence of CAD-induced structural alterations of the arterial wall ${ }^{24}$ that might have contributed to the manifestation of the MBF heterogeneity during CPT. Second, because the sample size of the study population was relatively small, larger prospective investigations in patients without or with diffuse $\mathrm{CAD}$ are desirable to draw more definite conclusions. Finally, as we did not assess the hyperemic MBF increases to pharmacologic vasodilation, which is clinically more widely used, further prospective angiographic studies are necessary to investigate the diagnostic accuracy of the heterogeneity in longitudinal MBF during pharmacologic vasodilation to identify an impairment of flow-mediated coronary vasomotor function.

\section{Conclusions}

Although the assessment of a heterogeneity in longitudinal MBF during CPT appears to be more sensitive than the CPT-induced change in global MBF in identifying coronary vasomotor dysfunction, the combined analysis of both parameters yields the highest diagnostic accuracy of the noninvasive evaluation of coronary vasomotor function. Combining both quantitative measures of MBF responses to CPT may emerge as a new promising analytic approach to further optimize the noninvasive identification and characterization of coronary vasomotor dysfunction.

\section{Acknowledgment}

We are indebted to the nurses of the Cardiac Catheterization and PET-Cyclotron Unit of Albert Ludwig University, Freiburg, Germany, for their invaluable support.

The authors have indicated they have no financial conflicts of interest.

\section{References}

1. Schindler TH, Nitzsche EU, Olschewski M, Brink I, Mix M, Prior $\mathrm{J}$, et al. PET-measured responses of MBF to cold pressor testing correlate with indices of coronary vasomotion on quantitative coronary angiography. J Nucl Med 2004;45:419-28.

2. Schindler TH, Cardenas J, Prior JO, Facta AD, Kreissl MC, Zhang $\mathrm{XL}$, et al. Relationship between increasing body weight, insulin resistance, inflammation, adipocytokine leptin, and coronary circulatory function. J Am Coll Cardiol 2006;47:1188-95. 
3. Schindler TH, Nitzsche EU, Munzel T, Olschewski M, Brink I, Jeserich $\mathrm{M}$, et al. Coronary vasoregulation in patients with various risk factors in response to cold pressor testing: contrasting myocardial blood flow responses to short- and long-term vitamin C administration. J Am Coll Cardiol 2003;42:814-22.

4. Prior JO, Quinones MJ, Hernandez-Pampaloni M, Facta AD, Schindler TH, Sayre JW, et al. Coronary circulatory dysfunction in insulin resistance, impaired glucose tolerance, and type 2 diabetes mellitus. Circulation 2005;111:2291-8.

5. Schindler TH, Nitzsche EU, Olschewski M, Magosaki N, Mix M, Prior JO, et al. Chronic inflammation and impaired coronary vasoreactivity in patients with coronary risk factors. Circulation 2004;110:1069-75.

6. Campisi R, Czernin J, Schoder H, Sayre JW, Marengo FD, Phelps $\mathrm{ME}$, et al. Effects of long-term smoking on myocardial blood flow, coronary vasomotion, and vasodilator capacity. Circulation 1998; 98:119-25.

7. Di Carli MF, Bianco-Batlles D, Landa ME, Kazmers A, Groehn H, Muzik O, et al. Effects of autonomic neuropathy on coronary blood flow in patients with diabetes mellitus. Circulation 1999;100: 813-9.

8. Kaufmann PA, Camici PG. Myocardial blood flow measurement by PET: technical aspects and clinical applications. J Nucl Med 2005;46:75-88.

9. Widlansky ME, Gokce N, Keaney JF Jr, Vita JA. The clinical implications of endothelial dysfunction. J Am Coll Cardiol 2003; 42:1149-60

10. Ganz P, Vita JA. Testing endothelial vasomotor function: nitric oxide, a multipotent molecule. Circulation 2003;108:2049-53.

11. Schindler TH, Nitzsche EU, Schelbert HR, Olschewski M, Sayre J, Mix M, et al. Positron emission tomography-measured abnormal responses of myocardial blood flow to sympathetic stimulation are associated with the risk of developing cardiovascular events. J Am Coll Cardiol 2005;45:1505-12.

12. Lerman A, Zeiher AM. Endothelial function: cardiac events. Circulation 2005;111:363-8.

13. Fichtlscherer S, Breuer S, Zeiher AM. Prognostic value of systemic endothelial dysfunction in patients with acute coronary syndromes: further evidence for the existence of the "vulnerable" patient. Circulation 2004;110:1926-32.

14. Modena MG, Bonetti L, Coppi F, Bursi F, Rossi R. Prognostic role of reversible endothelial dysfunction in hypertensive postmenopausal women. J Am Coll Cardiol 2002;40:505-10.

15. Schindler TH, Facta AD, Prior JO, Cadenas J, Hsueh W, Quinones M, et al. Improvement of coronary vascular dysfunction in type 2 diabetic patients with euglycemic control. Heart 2007; $93: 345-9$.

16. Quinones MJ, Hernandez-Pampaloni M, Schelbert H, BulnesEnriquez I, Jimenez X, Hernandez G, et al. Coronary vasomotor abnormalities in insulin-resistant individuals. Ann Intern Med 2004; 140:700-8.

17. Cecchi F, Olivotto I, Gistri R, Lorenzoni R, Chiriatti G, Camici PG. Coronary microvascular dysfunction and prognosis in hypertrophic cardiomyopathy. N Engl J Med 2003;349:1027-35.

18. Sdringola S, Loghin C, Boccalandro F, Gould KL. Mechanisms of progression and regression of coronary artery disease by PET related to treatment intensity and clinical events at long-term follow-up. J Nucl Med 2006;47:59-67.

19. Gould KL. Assessing progression or regression of CAD: the role of perfusion imaging. J Nucl Cardiol 2005;12:625-38.

20. Zeiher AM, Drexler H. Coronary hemodynamic determinants of epicardial artery vasomotor responses during sympathetic stimulation in humans. Basic Res Cardiol 1991;86(Suppl 2):203-13.

21. Zeiher AM, Drexler H, Saurbier B, Just H. Endothelium-mediated coronary blood flow modulation in humans. Effects of age, atherosclerosis, hypercholesterolemia, and hypertension. J Clin Invest 1993;92:652-62

22. Zeiher AM, Drexler H, Wollschlager H, Just H. Modulation of coronary vasomotor tone in humans. Progressive endothelial dysfunction with different early stages of coronary atherosclerosis. Circulation 1991;83:391-401.

23. el-Tamimi H, Mansour M, Pepine CJ, Wargovich TJ, Chen H. Circadian variation in coronary tone in patients with stable angina. Protective role of the endothelium. Circulation 1995;92:3201-5.

24. Gould KL, Nakagawa Y, Nakagawa K, Sdringola S, Hess MJ, Haynie M, et al. Frequency and clinical implications of fluid dynamically significant diffuse coronary artery disease manifest as graded, longitudinal, base-to-apex myocardial perfusion abnormalities by noninvasive positron emission tomography. Circulation 2000;101:1931-9.

25. Gould KL, Martucci JP, Goldberg DI, Hess MJ, Edens RP, Latif $\mathrm{R}$, et al. Short-term cholesterol lowering decreases size and severity of perfusion abnormalities by positron emission tomography after dipyridamole in patients with coronary artery disease. A potential noninvasive marker of healing coronary endothelium. Circulation 1994;89:1530-8.

26. Hernandez-Pampaloni M, Keng FY, Kudo T, Sayre JS, Schelbert HR. Abnormal longitudinal, base-to-apex myocardial perfusion gradient by quantitative blood flow measurements in patients with coronary risk factors. Circulation 2001;104:527-32.

27. Schindler TH, Facta AD, Prior JO, Campisi R, Inubushi M, Kreissl $\mathrm{MC}$, et al. PET-measured heterogeneity in longitudinal myocardial blood flow in response to sympathetic and pharmacologic stress as a non-invasive probe of epicardial vasomotor dysfunction. Eur J Nucl Med Mol Imaging 2006;33:1140-9.

28. Wollschlaeger H, Lee P, Zeiher AM, Solzbach U, Just H. Improvement of quantitative angiography by exact calculation of radiological magnification factor. IEEE Comput Cardiol 1985:483-6.

29. Kuhle WG, Porenta G, Huang SC, Buxton D, Gambhir SS, Hansen $\mathrm{H}$, et al. Quantification of regional myocardial blood flow using $13 \mathrm{~N}$-ammonia and reoriented dynamic positron emission tomographic imaging. Circulation 1992;86:1004-17.

30. Zeiher AM, Drexler H, Wollschlaeger H, Saurbier B, Just H. Coronary vasomotion in response to sympathetic stimulation in humans: importance of the functional integrity of the endothelium. J Am Coll Cardiol 1989;14:1181-90.

31. Schindler TH, Zhang XL, Cadenas J, Sayre J, Dahlbom M, Schelbert HR. Assessment of intra- and interobserver reproducibility of rest and cold-pressor-test stimulated myocardial blood flow with 13N-ammonia and PET. Eur J Nucl Med Mol Biol 2007;34: 1178-88. Epub 2007 Mar 3.

32. Siegrist PT, Gaemperli O, Koepfli P, Schepis T, Namdar M, Valenta I, et al. Repeatability of cold pressor test-induced flow increase assessed with $\mathrm{H}(2)(15) \mathrm{O}$ and PET. J Nucl Med 2006;47: 1420-6.

33. Camici PG, Crea F. Coronary microvascular dysfunction. N Engl J Med 2007;356:830-40.

34. Schindler TH, Zhang XL, Vincenti G, Mhiri L, Lerch R, Schelbert $\mathrm{H}$. Role of PET in the evaluation and understanding of coronary physiology. J Nucl Cardiol. In press 2007.

35. Buus NH, Bottcher M, Hermansen F, Sander M, Nielsen TT, Mulvany MJ. Influence of nitric oxide synthase and adrenergic inhibition on adenosine-induced myocardial hyperemia. Circulation 2001;104:2305-10.

36. Tawakol A, Forgione MA, Stuehlinger M, Alpert NM, Cooke JP, Loscalzo J, et al. Homocysteine impairs coronary microvascular dilator function in humans. J Am Coll Cardiol 2002;40: 1051-8. 
37. Bonetti PO, Lerman LO, Lerman A. Endothelial dysfunction: a marker of atherosclerotic risk. Arterioscler Thromb Vasc Biol 2003;23:168-75.

38. Schachinger V, Zeiher AM. Prognostic implications of endothelial dysfunction: does it mean anything? Coron Artery Dis 2001;12: 435-43.

39. De Bruyne B, Hersbach F, Pijls NH, Bartunek J, Bech JW, Heyndrickx GR, et al. Abnormal epicardial coronary resistance in patients with diffuse atherosclerosis but "normal" coronary angiography. Circulation 2001;104:2401-6.

40. Sdringola S, Patel D, Gould KL. High prevalence of myocardial perfusion abnormalities on positron emission tomography in asymptomatic persons with a parent or sibling with coronary artery disease. Circulation 2001;103:496-501.

41. Kern MJ. Coronary physiology revisited: practical insights from the cardiac catheterization laboratory. Circulation 2000;101:1344-51.
42. Drexler H, Zeiher AM, Wollschlager H, Meinertz T, Just H, Bonzel T. Flow-dependent coronary artery dilatation in humans. Circulation 1989;80:466-74.

43. Cox DA, Vita JA, Treasure CB, Fish RD, Alexander RW, Ganz P, et al. Atherosclerosis impairs flow-mediated dilation of coronary arteries in humans. Circulation 1989;80:458-65.

44. Victor RG, Leimbach WN Jr, Seals DR, Wallin BG, Mark AL. Effects of the cold pressor test on muscle sympathetic nerve activity in humans. Hypertension 1987;9:429-36.

45. Demaria AN, Ben-Yehuda O, Berman D, Feld GK, Ginsberg J, Greenberg BH, et al. Highlights of the year in JACC 2005. J Am Coll Cardiol 2006;47:184-202.

46. Nabel EG, Ganz P, Gordon JB, Alexander RW, Selwyn AP. Dilation of normal and constriction of atherosclerotic coronary arteries caused by the cold pressor test. Circulation 1988;77: 43-52.

\section{ON THE MOVE? \\ Send us your new address at least six weeks ahead}

Don't miss a single issue of the journal! To ensure prompt service when you change your address, please photocopy and complete the form below.

Please send your change of address notification at least six weeks before your move to ensure continued service. We regret we cannot guarantee replacement of issues missed due to late notification.

\section{JOURNAL TITLE:}

Fill in the title of the journal here.

\section{OLD ADDRESS:}

Affix the address label from a recent issue of the journal here.

\section{NEW ADDRESS: \\ Clearly print your new address here.}

Name

Address

City/State/ZIP

\section{COPY AND MAIL THIS FORM TO:}

Elsevier Inc.

Subscription Customer Service

6277 Sea Harbor Dr

Orlando, FL, 32887
OR FAX TO:

407-363-9661
OR PHONE:

800-654-2452

Outside the US, call 407-345-4000 